\title{
Adverse life events, area socioeconomic disadvantage, and psychopathology and resilience in young children: the importance of risk factors' accumulation and protective factors' specificity
}

\author{
Eirini Flouri $\cdot$ Nikos Tzavidis $\cdot$ Constantinos Kallis
}

Received: 6 March 2009/Accepted: 21 September 2009/Published online: 10 October 2009

(C) Springer-Verlag 2009

\begin{abstract}
Few studies on resilience in young children model risk appropriately and test theory-led hypotheses about its moderation. This study addressed both issues. Our hypothesis was that for preschool children's emotional/ behavioral adjustment in the face of contextual risk protective factors should be located in the cognitive domain. Data were from the first two sweeps of the UK's Millennium Cohort Study. The final study sample was 4,748 three-year-old children clustered in 1,549 Lower layer Super Output Areas in nine strata. Contextual risk was measured at both area (with the Index of Multiple Deprivation) and family (with proximal and distal adverse life events experienced) level. Moderator variables were parenting, verbal and non-verbal ability, developmental milestones, and temperament. Multivariate multilevel models-that allowed for correlated residuals at both individual and area level-and univariate multilevel models estimated risk effects on specific and broad psychopathology. At baseline, proximal family risk, distal family risk and area risk were all associated with broad psychopathology, although the most parsimonious was the
\end{abstract}

\footnotetext{
E. Flouri $(\bowtie)$

Department of Psychology and Human Development, Institute of Education, University of London, 25 Woburn Square, London WC1H 0AA, UK

e-mail: e.flouri@ioe.ac.uk

N. Tzavidis

Social Statistics and Centre for Census and Survey Research, University of Manchester, Manchester, UK

e-mail: nikos.tzavidis@manchester.ac.uk

\section{Kallis}

London School of Hygiene and Tropical Medicine,

University of London, London, UK

e-mail: Constantinos.Kallis@1shtm.ac.uk
}

proximal family risk model. The area risk/broad psychopathology association remained significant even after family risk was controlled but not after family level socioeconomic disadvantage was controlled. The cumulative family risk was more parsimonious than the specific family risks model. Non-verbal ability moderated the effect of proximal family risk on conduct and emotional problems, and developmental milestones moderated the effect of proximal family risk on conduct problems. The findings highlight the importance of modeling contextual risk appropriately and of locating in the cognitive domain factors that buffer its effect on young children's adjustment.

Keywords Hierarchical data $\cdot$ MCS $\cdot$ Multilevel models . Multivariate multilevel models · Psychopathology . Resilience $\cdot$ Strengths and Difficulties Questionnaire

\section{Introduction}

In the classic approach to cumulative contextual risk in child psychiatry [1], organismic characteristics as well as proximal and distal qualities of the environment are modeled collectively. For each environmental construct, a dichotomous classification of risk exposure is determined, typically by a statistical cut off (e.g., greater than one standard deviation above the mean, upper quartile, etc.) or on the basis of a conceptual categorization (e.g., being below the poverty line). Cumulative risk is then calculated by a simple summation of the multiple risk categories. These risk categories are usually not weighted. In this approach, therefore, risk is viewed as an accumulation of stressors, and the number of risks that children experience carries more gravity than the experience of any particular 
risk. Indeed, cumulative risk indexes have been noted for their potential to capture the natural covariation of risk factors. For example, physical risk factors, such as poor housing quality, noise and pollution are strongly interrelated as are psychosocial risk parameters such as family turmoil and violence [2]. Furthermore, aggregate variables of risk are more stable than any individual measure, and there is increased power to detect effects because errors of measurement decrease as scores are summed and degrees of freedom are preserved [3]. Cumulative risk measures are consistently found to explain more variance in children's outcomes than single factors [4-7].

Despite this, few studies [8] have examined the relationship between multiple risk exposure and young children's psychopathology using a cumulative risk approach. In fact, even studies with school-aged children have yet to deal with several issues with respect to the modeling of cumulative risk. First, studies have yet to establish convincingly the effect of the timing of cumulative contextual risk on psychopathology $[4,9,10]$. Second, with few exceptions [4, 11-14], studies do not examine the functional form of cumulative contextual risk's effect on psychopathology. There is evidence for a linear effect whereby increments in risk factors have a steady, additive impact on mental health problems in school-aged children [5]. However, as few researchers actually report whether their investigations included appropriate tests for nonlinear patterns of cumulative risk, this ignores the possibility of a nonlinear relationship that might manifest itself as an acceleration [14] or a leveling-off [13] of problems at a critical level of risk. Third, despite the renewed interest in neighborhood effects on children's development [15], only few studies [16] compare family and neighborhood risk. Fourth, although it is possible that the effect of one type or one level of risk on psychopathology is conditional upon the value of another, interactions between types and levels of risk on child psychopathology are not usually studied. Fifth and finally, with few exceptions [4, 8, 17, 18], most studies do not examine factors that protect from negative outcomes in children exposed to cumulative contextual risk. The dearth of such research is unfortunate as various protective factors have been identified as moderating the impact of specific contextual risk. These factors are grouped under two domains, namely, individual attributes and connections to competent and caring adults in the family and the community [19, 20].

\section{Moderation of risk}

To the best of our knowledge, the only study modeling the moderation of cumulative contextual risk in preschool children's emotional and behavioral adjustment [8] found that low vagal tone during tasks protected from the effect of multiple risks, and concluded that this is because low vagal tone during tasks may reflect regulatory capacities that allow children to engage with learning opportunities. This suggests that children's advanced cognitive development may be the factor that moderates the effect of contextual risk on emotional and behavioral outcomes. Although previous research has certainly located in the cognitive domain protective factors for school-aged children's emotional and behavioral adjustment in the face of cumulative contextual risk [4, 18], studies have yet to establish the importance of cognitive protective factors for preschool children's adjustment in the face of cumulative contextual risk or test the hypothesis that for preschool children's adjustment in the face of cumulative contextual risk factors promoting resilience should be located in the cognitive rather than the biological or the social domain.

This study was undertaken to meet this aim, i.e., to test that for preschool children's adjustment in the face of cumulative contextual risk factors promoting resilience should be located in the cognitive domain. In doing so, it also extended in several ways prior work on the role of contextual risk in child psychopathology. First, it used items from a well-validated measure of multiple family risks. However, since only a selection of the original items could be used, it also tested appropriately for the effect or risk accumulation and risk specificity when modeling the effect of family risk on child psychopathology. Second, it measured both distal and proximal family risk to test for the effect of the timing of risk on psychopathology. Third, it measured area risk and compared area with family risk effects. Fourth, it searched for an appropriate functional form of both area and family risk's effect on psychopathology. Fifth and finally, it tested for the presence of interaction effects between proximal, distal and area risk on psychopathology.

\section{Method}

Participants and procedure

Data were obtained from the first two sweeps of the Millennium Cohort Study (MCS), a longitudinal survey drawing its sample from all live births in the UK over a period of 12 months, beginning on 1 September 2000 in England and Wales, and 3 months later in Scotland and Northern Ireland. The sample was drawn slightly later in Scotland and Northern Ireland so as not to coincide with other surveys being carried out on families with babies in these areas at the same time. Sweep 1 took place when the children were aged 9 months, and Sweep 2 took place when the children were around 3 years. The sample design 
allowed for disproportionate representation of families living in areas of child poverty in Northern Ireland, Scotland and Wales and in areas with high ethnic minority populations in England. In all there were nine strata: England-advantaged, England-disadvantaged, England-ethnic, Wales-advantaged, Wales-disadvantaged, Scotland-advantaged, Scotland-disadvantaged, Northern Ireland-advantaged, and Northern Ireland-disadvantaged. Data on child psychopathology were collected at Sweep 2 from the main respondent. Although the MCS is a study of 19,244 children, there were complete data on broad psychopathology for a total of 9,736 children clustered in Lower layer Super Output Areas (LSOAs). LSOAs are built from groups of Output Areas (typically 4-6), and are constrained by the boundaries of the Standard Table wards used for 2001 Census outputs. They have, on average, 1,500 residents. For example, there are 175,434 Output Areas and 34,378 LSOAs in England and Wales. In this study, LSOAs, rather than the electoral wards on which the MCS survey design was built, were used to get smaller 'neighborhoods' and more up-to-date ecological correlates. For one child, there was no information on the LSOA at Sweep 2 and, therefore, this case was removed from our analysis.

\section{Measures}

Area contextual risk was measured with the Index of Multiple Deprivation (IMD), a weighted area level aggregation of specific dimensions of deprivation. The dimensions of deprivation used to construct the English IMD 2004, for instance, were (1) income deprivation, (2) employment deprivation, (3) health deprivation and disability, (4) education, skills and training deprivation, (5) barriers to housing and services, (6) living environment deprivation and (7) crime [21]. As the various UK countries' indices of multiple deprivation are not comparable, IMD ranks were used, which for the purposes of this study were standardized. As discussed above, the geography we worked with was at LSOA level and the IMD score was measured at LSOA level. Many families changed LSOAs between Sweeps 1 and 2 (of the 19,244 cohort members, $52.4 \%$ did not change LSOA between Sweeps 1 and 2, 44\% did, and for $3.6 \%$ there was missing information about LSOA at Sweep 1), but most remained within the same LSOA. The IMD scores at Sweeps 1 and 2 were highly correlated (the correlation between the IMD ranks at Sweeps 1 and 2 was 0.911), and we, therefore, used IMD at Sweep 1 in all our analyses.

Distal (birth to Sweep 1) and proximal (Sweep 1 to Sweep 2) family contextual risk was measured with a subset of Tiet et al.'s [18] Adverse Life Events Scale. This scale is composed of 25 possible events for which children had little or no control over and is a modification of the
Life Events Checklist (LEC) [22-24], a psychometrically valid [22] measure of exposure to potentially traumatic events developed at the National Center for Posttraumatic Stress Disorder (PTSD) to facilitate the diagnosis of PTSD. The self-report version of the Adverse Life Events Scale has been used with children as young as 9 years [25], and measures exposure to adverse life events at both family (e.g., 'negative change in parents' financial situation'), and child (e.g., 'got seriously ill or injured', 'lost a close friend') levels. In view of this study's research aims the version of the scale used comprised items which (a) measured only family level risk, (b) were developmentally appropriate, (c) could be reconstructed from the MCS data, and (d) measured both distal and proximal risk. In all, information about eight adverse life events was available both between birth and Sweep 1 ('distal family risk') and between Sweeps 1 and 2 ('proximal family risk') in MCS. The eight events corresponded to the following Adverse Life Events Scale items: 'family member died', 'family member was seriously injured', 'negative change in parents' financial situation', 'family member had mental/emotional problem', 'family moved', 'got a new brother or sister', 'one of the parents went to jail', and 'parents separated'. In contrast to the high correlation in IMD scores at Sweeps 1 and 2, the correlation between family risk at Sweep 1 and family risk at Sweep 2 was 0.201 .

Broad and specific psychopathology was assessed with the main caregiver's report of the Strengths and Difficulties Questionnaire (SDQ), a 25-item 3-point (ranging from 0 to 2) scale measuring four difficulties (hyperactivity, emotional symptoms, conduct problems, and peer problems), as well as prosocial behavior [26, 27]. Each subscale has five items. A total difficulties (broad psychopathology) scale is calculated by summing the scores for hyperactivity, emotional symptoms, conduct problems and peer problems (http://www.sdqinfo.com). The SDQ has both good testretest reliability [28] and excellent concurrent and discriminant validity $[29,30]$.

The child level covariates were age and sex, and the family level covariates, all measured at Sweep 1, were family structure, maternal psychological distress, and, to index family's socioeconomic status, maternal social class and qualifications. Maternal psychological distress was measured with nine items from the Malaise Inventory [31], a psychometrically valid [32] measure of depressed mood. Maternal social class, measured with the National Statistics Socio-economic Classification (NS-SEC), ranged from 1 ('high managerial'/'professional') to 7 ('routine'). Mothers' highest qualifications were grouped into six major categories, ranging from 1 to 6 ('level 5', i.e., first/higher degree), roughly equivalent to National Vocational Qualification (NVQ) levels. 
The moderator variables examined were developmental milestones, temperament and parenting, all assessed in Sweep 1 by the main respondent, and verbal and nonverbal ability, measured in Sweep 2. Developmental milestones are a set of functional skills or age-specific tasks that most children can do at a certain age range. In the MCS developmental milestones assessing communicative gestures as well as fine and gross motor coordination typical for a 9-month-old child were measured with 12 3-point scales from the Denver Developmental Screening Test [33], the most popular tool to screen for potential developmental problems. A high score on the test indicated developmental delay. Temperament or individual differences in reactivity and self-regulation that are assumed to have a relatively enduring, biological basis, was measured in the MCS with items selected from the Carey Infant Temperament Scale [34, 35], used also in the Avon Longitudinal Study of Parents and Children (ALSPAC) and the National Longitudinal Survey of Youth. The items included in the MCS measured three dimensions of temperament, namely mood (measured with five items such as is pleasant'), adaptability (measured with five items such as 'is rarely or almost never wary of strangers') and regularity (measured with four items such as 'gets sleepy at about the same time'). Parenting was measured with four 5-point scales, originally derived from the European Longitudinal Study of Pregnancy and Childhood, and used in other UK longitudinal studies (such as the ALSPAC), assessing to what extent the main respondent agreed that it is important to develop a regular pattern of feeding and sleeping with a baby', 'babies need to be stimulated if they are to develop well', 'talking, even to a young baby, is important', and 'cuddling a baby is very important'. The four-item scale for the whole MCS at Sweep 1 yielded a Cronbach's alpha of 0.67 , with a high scale score indicating negligent parenting. Finally, verbal and non-verbal ability were measured with the naming vocabulary subtest of the British Ability Scales (BAS) and with the School Readiness Composite (SRC) of the Revised Bracken Basic Concept Scale, respectively. The BAS Naming Vocabulary subtest measures children's expressive language skills, and the SRC measures 'readiness' for formal education. Both assessments were individually administered in Sweep 2 by survey team members in computer-assisted interviews. The BAS Naming Vocabulary subtest is part of a cognitive test battery designed for children aged 3-17 [36]. Children are asked to name a series of pictures of everyday items. There are 36 items in total, and the assessment is terminated if five successive items are answered incorrectly. In the MCS, the test was not administered to children who did not speak English. The SRC comprises six subtests of the Revised Bracken Basic Concept Scale measuring children's knowledge of those 'readiness' concepts that parents and teachers traditionally teach children in preparation for formal education [37]. The test has been designed for children in the age range of 2.5-7 years and 11 months. The six subtests of the SRC comprise the assessment of children's basic concepts of colors, letters, numbers/ counting, sizes, comparisons and shapes.

\section{Models}

The structure of the MSC data with children clustered within LSOAs dictates the use of statistical models that appropriately account for the nested structure. One class of statistical models that provide valid inferences in the case of hierarchical data is multilevel random effects models [38]. To be consistent with the hierarchical structure of the MCS data, we describe multilevel models using a two-level structure under which children are clustered within LSOAs at Sweep 2. The simplest two-level model for total difficulties is a random intercepts model described by

$y_{i j}=x_{i j}^{T} \beta+u_{j}+\varepsilon_{i j}, \quad i=1, \ldots, n, \quad j=1, \ldots, d$

where $y_{i j}$ is the response variable (total difficulties) for child $i$ in LSOA $j, x_{i j}$ denotes a set of explanatory variables that can be defined at child, family and LSOA level, $u_{j}$ denotes a vector of random effects associated with LSOA $j$ and $\varepsilon_{i j k}$ denotes the level 1 (child level) residual term.

As information for each child was available for each of the five SDQ subscales, allowing for a different model for each SDQ subscale may provide a deeper insight. However, it is possible that responses to the different SDQ subscales are correlated. To account for the existence of correlation in each child's responses, we fitted multivariate response multilevel models that allowed for the error terms of the different models to be correlated. Let us denote by $i$ the subscript referring to the child, by $j$ the subscript referring to the LSOA, and by $l$ the subscript denoting a specific SDQ subscale. The multivariate response multilevel model is then defined as

$$
\begin{aligned}
& y_{i j l}=x_{i j l}^{T} \beta_{l}+u_{j l}+\varepsilon_{i j l}, \quad i=1, \ldots, n, \quad j=1, \ldots, d, \\
& \quad l=1, \ldots, 5
\end{aligned}
$$

where $\varepsilon_{i j l} \sim N\left(0, \Sigma_{\varepsilon}\right)$ with $\Sigma_{\varepsilon}$ denoting the variance covariance matrix between the level 1 error terms and $u_{j l} \sim N\left(0, \Sigma_{u}\right)$ with $\Sigma_{u}$ denoting the variance covariance matrix between the level 2 error terms. Modeling specific psychopathologies in a multivariate way offers a more flexible modeling framework as it can accommodate different covariates for the different SDQ subscales as well as allow for the correlation between unobserved factors affecting the scores on the different SDQ subscales at different hierarchical levels. When compared with a single multilevel model for broad psychopathology, a multivariate 
multilevel model offers an additional advantage with respect to the handling of missing data. In particular, when modeling broad psychopathology, a missing value in one of the items comprising total difficulties on the SDQ results in a missing total difficulties value. This is not the case with multivariate models, since missing values in some of the SDQ subscales do not result in the omission of the corresponding unit from the analysis. In this way, the estimates obtained from the multivariate multilevel model are efficient.

The complex survey design of the MCS was accounted for by conditioning on the design variables, i.e., the variables used in designing the MCS survey. Therefore, in the models described below, stratification was accounted for by including a set of dummy variables representing the nine strata of the MCS, whereas clustering was accounted for by running multilevel models. The models assumed that data were missing at random (MAR) [38].

\section{Results}

We used STATA version 10 and MLwiN to run random intercepts models (see Table 1 for a model summary), described by (1), allowing the constant to vary at level 2 , i.e., LSOA at Sweep 2. We first estimated an empty model for total difficulties in the 9,735 children with valid data on total difficulties. This model included only the design variables to adjust for the effects of stratification. The two-level empty model showed that the average total difficulties score as reflected in the intercept was 8.219 $(\mathrm{SE}=0.102)$. The child level variance component (level 1 variance) was $23.367(\mathrm{SE}=0.353)$, and the variance due to differences in LSOAs was 0.850 ( $\mathrm{SE}=0.166$ ). This suggests a significant between LSOA variation. Using the two variance components to partition the variance across levels we found that the intra-cluster correlation coefficient was 0.035 . This suggests that controlling only for stratification $3.5 \%$ of the variance in total difficulties scores was attributable to the area level. Therefore, although small the intra-cluster correlation justified the use of hierarchical modeling. We first investigated the contextual risk/total difficulties association by running three baseline models, one with proximal family risk, one with distal family risk and one with area risk. Area risk $(b=-0.938, \mathrm{SE}=0.083)$, distal family risk $(b=0.852$, $\mathrm{SE}=0.060)$, and proximal family risk $(b=0.715$, $\mathrm{SE}=0.043)$ were all related to total difficulties. As expected, the amount of variance due to differences in areas explained in this model when area risk was added to
Table 1 Model summary (broad psychopathology)

\footnotetext{
a The design variables used were the MCS strata defined by country (England, Scotland, Wales and Northern Ireland) and disadvantage status according to the Child Poverty Index (CPI). For England, there was an additional stratum, i.e. ethnic minority indicating wards which, in the 1991 Census of Population, had an ethnic minority indicator of at least $30 \%$. In other words, at least $30 \%$ of their total population fell into the categories 'Black' or 'Asian'
}

\begin{tabular}{|c|c|}
\hline Models & Specification \\
\hline Model 1 & Design variables ${ }^{\mathrm{a}}$ \\
\hline Model 2 & Model $1+$ area random effect \\
\hline Model 3.1 & Model $2+$ proximal family risk \\
\hline Model 3.2 & Model $2+$ distal family risk \\
\hline Model 3.3 & Model $2+$ area risk \\
\hline Model 4 & Model $2+$ area risk + measures of family risk (proximal and distal family risk) \\
\hline Model 5 & $\begin{array}{l}\text { Model } 4+\text { family level fixed effects (family structure, mother's qualifications, } \\
\text { mother's social class and mother's psychological distress) }\end{array}$ \\
\hline Model 6 & $\begin{array}{l}\text { Model } 5+\text { child level fixed effects (gender, age) }+ \text { moderator variables (mood, } \\
\text { regularity, adaptability, non-verbal ability, verbal ability, developmental delay, } \\
\text { negligent parenting) }\end{array}$ \\
\hline Model 7.1 & Model $6+$ quadratic effect for area risk \\
\hline Model 7.2 & Model $6+$ quadratic effect for distal family risk \\
\hline Model 7.3 & Model $6+$ quadratic effect for proximal family risk \\
\hline Model 8.1 & Model $6+$ interaction between proximal family risk and distal family risk \\
\hline Model 8.2 & Model $6+$ interaction between proximal family risk and area risk \\
\hline Model 8.3 & Model $6+$ interaction between distal family risk and area risk \\
\hline Model 9.1 & Model $6+$ interaction between proximal family risk and mood \\
\hline Model 9.2 & Model $6+$ interaction between proximal family risk and regularity \\
\hline Model 9.3 & Model $6+$ interaction between proximal family risk and adaptability \\
\hline Model 9.4 & Model $6+$ interaction between proximal family risk and non-verbal ability \\
\hline Model 9.5 & Model $6+$ interaction between proximal family risk and verbal ability \\
\hline Model 9.6 & Model $6+$ interaction between proximal family risk and developmental delay \\
\hline Model 9.7 & Model $6+$ interaction between proximal family risk and parenting \\
\hline
\end{tabular}


the empty model was reduced $(0.697, \mathrm{SE}=0.152)$. To compare the goodness of fit of these three baseline models, we used an appropriate statistic that takes into account that the models are not nested. Using the Bayesian Information Criterion (BIC), a function of the likelihood, the number of observations and the number of free parameters of each model [39], we found that the most parsimonious model was the proximal family risk model. The association of area risk, distal family risk and proximal family risk with total difficulties remained significant even when all three risk variables were entered in the same model. The amount of variance due to differences in areas explained in this model was further reduced $(0.606, \mathrm{SE}=0.140)$, but was still significant. This suggests that although child psychopathology scores differed by area partly because of differences in area level socioeconomic disadvantage, even with area socioeconomic disadvantage adjusted there was still area level variance that remained unexplained.

In the next step, the family level variables of family structure, and mother's qualifications, social class and psychological distress were added. With these variables in the model, the effect of area risk on total difficulties became nonsignificant. The amount of variance in total psychopathology scores due to differences in area explained in the model became nonsignificant $(0.090$, $\mathrm{SE}=0.093)$. Taken together, these two findings suggest that both the area differences in total difficulties and the effect of area risk on total difficulties operated via family characteristics. The effect of distal and proximal family risk, on the other hand, remained significant $(b=0.238$, $\mathrm{SE}=0.065$, and $b=0.376, \mathrm{SE}=0.044$, respectively) suggesting that family contextual risk predicts child psychopathology directly and independently of family structure, socioeconomic status or maternal psychopathology.

Next, the full model (Model 6) was introduced. This added the child level control variables and the proposed moderators. The final study sample obtained after omitting cases with missing values on both broad psychopathology and all its predictors was 4,748 children clustered in 1,549 LSOAs. The number of children per LSOA ranged from 1 to 41 , with an average of 3.1 children per LSOA. The distribution of the 4,748 children in the nine MCS strata in Sweep 2 was as follows: England-advantaged: 1,847; England-disadvantaged: 884; England-ethnic: 139; Walesadvantaged: 319; Wales-disadvantaged: 421; Scotlandadvantaged: 434; Scotland-disadvantaged: 264; Northern Ireland-advantaged: 220, and Northern Ireland-disadvantaged: 220. As can be seen in Table 2, although negligent parenting was marginally significant, all the child level moderator variables were statistically significant in predicting broad psychopathology. In this final model, the child level variance component (level 1 variance) was
$16.409(\mathrm{SE}=0.359)$, and the variance due to differences in LSOAs was $0.203(\mathrm{SE}=0.133)$.

As explained above, the initial MCS sample is 19,244 children. Of these, 9,736 had information about broad psychopathology, our main response variable, and of these, 4,748 children were the final study sample. As expected, these 4,748 children differed from the 14,496 children not included in the final study sample in most of the study variables. In particular, with regards to differences in Sweep 1 variables, compared with their counterparts those in the final study sample tended to live in less disadvantaged areas, in intact families, and with mothers who were less negligent, less distressed, more educated and of higher social class. With regards to differences in Sweep 2 variables, compared with their counterparts those in the study sample tended to present with less broad psychopathology, be female, have easier temperament, higher verbal and non-verbal cognitive ability, and have less developmental delay (all differences were statistically significant at $P<0.001$; results available from the authors). However, there was no difference between the two groups in age or in number of either proximal or distal family adverse life events experienced.

Comparing cumulative and specific family risk specifications

We could not compare the proximal cumulative risk specification with the proximal specific risk specification as one item of the proximal Adverse Life Events Scale ('one of the parents went to jail') was dropped due to collinearity. However, the comparison of the distal cumulative with the distal specific risk specification showed that, after applying a Bonferroni correction (alpha of $0.05 / 8$ adverse events $=0.00625$ ), none of the eight specific distal risks were significantly associated with broad psychopathology. The only distal risk item approaching significance was 'negative change in parents' financial situation' $(b=0.421, \mathrm{SE}=0.158)$. As the BIC for the cumulative risk model $(27,176.89)$ was lower than that for the specific risk model $(27,216.41)$, we concluded that the cumulative risk model specification should be preferred, and therefore this risk specification was used for the remaining analysis.

Investigating the appropriate functional form of contextual risk's effect and testing for moderator effects

We further investigated the appropriate functional form of contextual risk's effect on broad psychopathology by introducing quadratic terms for contextual risk separately in the full model (Model 6). None of the three quadratic 
Table 2 Broad psychopathology

\begin{tabular}{|c|c|c|c|c|c|c|}
\hline \multirow[t]{2}{*}{ Predictors } & \multicolumn{2}{|l|}{ Model 2} & \multicolumn{2}{|l|}{ Model 4} & \multicolumn{2}{|l|}{ Model 6} \\
\hline & Coeff. & SE & Coeff. & SE & Coeff. & SE \\
\hline Constant & 8.219 & 0.102 & 7.250 & 0.174 & 13.749 & 1.371 \\
\hline \multicolumn{7}{|c|}{ Stratum (Ref: England-advantaged) } \\
\hline England-disadvantaged & 1.927 & 0.170 & 0.849 & 0.186 & 0.315 & 0.208 \\
\hline England-ethnic & 2.732 & 0.289 & 1.983 & 0.294 & 0.630 & 0.405 \\
\hline Wales-advantaged & -0.155 & 0.257 & -1.609 & 0.289 & -0.528 & 0.321 \\
\hline Wales-disadvantaged & 1.276 & 0.224 & -0.406 & 0.266 & 0.021 & 0.302 \\
\hline Scotland-advantaged & -0.357 & 0.234 & -1.595 & 0.257 & -0.178 & 0.281 \\
\hline Scotland-disadvantaged & 1.115 & 0.268 & -0.403 & 0.293 & 0.188 & 0.334 \\
\hline Northern Ireland-advantaged & -0.715 & 0.286 & -2.142 & 0.318 & -0.844 & 0.360 \\
\hline Northern Ireland-disadvantaged & 1.194 & 0.260 & -0.435 & 0.298 & -0.854 & 0.360 \\
\hline Standardized IMD rank & & & -0.776 & 0.081 & -0.039 & 0.095 \\
\hline Proximal adverse life events & & & 0.581 & 0.044 & 0.394 & 0.054 \\
\hline Distal adverse life events & & & 0.666 & 0.061 & 0.308 & 0.079 \\
\hline \multicolumn{7}{|c|}{ Family structure (Ref: two natural parents) } \\
\hline Natural mother only & & & & & 0.260 & 0.208 \\
\hline Other & & & & & -0.959 & 1.828 \\
\hline \multicolumn{7}{|c|}{ Mother's NS-SEC (Ref: high managerial/professional) } \\
\hline Low managerial/professional & & & & & -0.047 & 0.229 \\
\hline Intermediate & & & & & 0.098 & 0.259 \\
\hline Small emp and self-employed & & & & & -0.824 & 0.361 \\
\hline Low supervisory and technical & & & & & 0.517 & 0.336 \\
\hline Semi routine & & & & & 0.728 & 0.275 \\
\hline Routine & & & & & 0.553 & 0.310 \\
\hline \multicolumn{7}{|c|}{ Mother's highest qualifications (Ref: level 5) } \\
\hline Level 4 & & & & & -0.426 & 0.306 \\
\hline Level 3 & & & & & 0.372 & 0.330 \\
\hline A/AS Level & & & & & 0.345 & 0.333 \\
\hline Level 2 & & & & & 0.920 & 0.314 \\
\hline Level 1 & & & & & 1.623 & 0.364 \\
\hline Mother's psychological distress & & & & & 0.472 & 0.040 \\
\hline Girl & & & & & -0.755 & 0.121 \\
\hline Age & & & & & 0.669 & $\mathbf{0 . 3 2 7}$ \\
\hline Mood & & & & & -0.153 & 0.019 \\
\hline Regularity & & & & & -0.134 & 0.023 \\
\hline Adaptability & & & & & -0.123 & 0.018 \\
\hline Non-verbal ability & & & & & -0.038 & 0.006 \\
\hline Verbal ability & & & & & -0.072 & 0.017 \\
\hline Developmental delay & & & & & 0.049 & $\mathbf{0 . 0 2 5}$ \\
\hline Negligent parenting & & & & & 0.073 & 0.042 \\
\hline Between area variability $\left(\sigma_{u}^{2}\right)$ & 0.849 & 0.155 & 0.606 & 0.133 & 0.171 & 0.135 \\
\hline Within area variability $\left(\sigma_{\varepsilon}^{2}\right)$ & 23.367 & 0.351 & 22.379 & 0.335 & 16.317 & 0.357 \\
\hline
\end{tabular}

terms for area, distal family, and proximal family risk were significant. We also tested if the effect of one type of risk was conditional upon the value of another. We entered these two-way interactions between distal, proximal and area risk variables separately in the full model, but neither interaction term was significant. This suggests that the effect of proximal family risk on broad psychopathology did not depend on the level of distal risk, and that area risk did not moderate the effect of either proximal or distal risk on broad psychopathology. 
We finally explored if parenting, verbal and non-verbal ability, developmental milestones, and mood, regularity and adaptability moderate the association between contextual risk and young children's broad psychopathology. As proximal risk was the type of risk more strongly associated with child psychopathology both at baseline and in the full model, interaction terms with proximal family risk and each of the proposed moderators were calculated and entered separately in the full model (Model 6). None of the interaction terms were significant, however, suggesting that the effect of proximal risk on broad psychopathology was not buffered either by child's easy temperament or advanced development, or by involved parenting. Table 2 presents the results of Model 6 and of the intermediate models (Models 2 and 4 ) that were of particular substantive significance.

\section{Multivariate response models}

We fitted multivariate response multilevel models described by [8] that allowed the error terms of the different models to be correlated and that included random area (LSOA) effects. These multivariate response two level models were effectively treated in MLwiN as three level models, i.e., with the responses as the additional lower level.

First, we ran an empty multivariate response multilevel model (Model 10). This model included only the design variables to adjust for the effects of stratification. The variance partition coefficients obtained showed that $1 \%$ of the total variation in prosocial behavior, $2 \%$ of the total variation in emotional symptoms, $2 \%$ of the total variation in conduct problems, $2 \%$ of the total variation in hyperactivity, and $2 \%$ of the total variation in peer problems were due to between LSOA variation.

We started our analysis by fitting three baseline models. The first model examined the effect of proximal family risk, which was significant in predicting scores in all five SDQ subscales. The second model examined the effect of distal risk, which was also significant in predicting scores in all five SDQ subscales. The third model examined the effect of area risk, which also had a significant, albeit weaker, effect on the four difficulties and a nonsignificant effect on prosocial behavior. The effect of proximal, distal and area risk on all four difficulties remained significant even when all risk variables were entered simultaneously (Model 13). However, the effect of distal risk on prosocial behavior became nonsignificant after adjusting for proximal and area risk suggesting that the effect of distal risk on prosocial behavior was via its impact on proximal risk.

Subsequently, we introduced the full model (Model 14). This added to the model including the design variables and the three risk variables all the family- and child level fixed effects, and all the moderator variables. The sample size in this full model was $28,672 .^{1}$ In this model, the effect of proximal risk on prosocial behavior became nonsignificant, as did the effect of area risk on all four difficulties. Although the effect of proximal risk remained significant on all four difficulties, the effect of distal risk was significant only on hyperactivity and conduct problems. Inspection of the between area and between children variance covariance matrices (Table 3) from the various models showed the covariances between the SDQ subscales at area level became less significant as we controlled for background characteristics. The covariances between the SDQ subscales at child level, however, remained significant even after controlling for a range of background characteristics in this final model (Model 14), which justifies the use of a multivariate model.

Next, we tested if the effect of one type of risk was conditional upon the value of another. We calculated twoway interactions between distal, proximal and area risk variables and entered these separately in the full model, but none of these interaction terms was significant. This suggests that the effect of proximal family risk on specific psychopathology did not depend on the level of distal risk, and that area risk did not moderate the effect of either proximal or distal risk on any specific child psychopathology.

Finally, we explored if parenting, verbal and non-verbal ability, developmental milestones, and mood, regularity and adaptability moderate the association between contextual risk and young children's specific psychopathology. As proximal risk was the type of risk more strongly associated with child psychopathology both at baseline and in the full model, interaction terms with proximal family risk and each of the proposed moderators were calculated and entered separately in the full model. The interaction between proximal family risk and developmental milestones predicted conduct problems $(b=-0.017$, $\mathrm{SE}=0.008)$, and the interaction between proximal family risk and non-verbal ability predicted both conduct problems $(b=-0.002, \mathrm{SE}=0.001)$ and emotional symptoms $(b=-0.002, \mathrm{SE}=0.001)$, but no other interaction effects were significant. These effects remained significant even when both interaction terms were entered in the same model (Model 17). These findings suggest that delayed development buffered the effect of proximal risk on conduct problems, and non-verbal ability buffered the effect of proximal risk on both emotional symptoms and conduct problems. Although developmental delay was not

\footnotetext{
1 This was the total number of observations in the multivariate (specific psychopathology) model. The number of observations for each SDQ outcome was as follows: Prosocial behavior: 5,719; Emotional symptoms: 5,966; Conduct problems: 5,954; Hyperactivity: 5,689, and Peer problems: 5,344.
} 
Table 3 Child level and area level covariance matrices between the five SDQ subscales estimated by fitting multivariate models (Models 10, 13, and 14)

\begin{tabular}{|c|c|c|c|c|c|}
\hline & Prosocial & Emotional & Conduct & Hyperactivity & Peer \\
\hline \multicolumn{6}{|l|}{ Model 10} \\
\hline \multicolumn{6}{|c|}{ Between area variance covariance matrix } \\
\hline Prosocial & $0.021(0.011)$ & & & & \\
\hline Emotional & $-0.011(0.007)$ & $0.042(0.008)$ & & & \\
\hline Conduct & $-0.018(0.010)$ & $0.050(0.009)$ & $0.088(0.017)$ & & \\
\hline Hyperactivity & $-0.018(0.011)$ & $0.048(0.010)$ & $0.080(0.016)$ & $0.090(0.022)$ & \\
\hline Peer & $-0.009(0.007)$ & $0.039(0.007)$ & $0.051(0.010)$ & $0.047(0.011)$ & $0.043(0.010)$ \\
\hline \multicolumn{6}{|c|}{ Between children variance covariance matrix } \\
\hline Prosocial & $3.302(0.043)$ & & & & \\
\hline Emotional & $-0.209(0.024)$ & $1.938(0.025)$ & & & \\
\hline Conduct & $-1.246(0.036)$ & $0.757(0.026)$ & $3.937(0.050)$ & & \\
\hline Hyperactivity & $-1.249(0.041)$ & $0.715(0.031)$ & $2.173(0.047)$ & $5.202(0.069)$ & \\
\hline Peer & $-0.759(0.027)$ & $0.611(0.020)$ & $0.719(0.029)$ & $0.745(0.033)$ & $2.105(0.029)$ \\
\hline \multicolumn{6}{|l|}{ Model 13} \\
\hline \multicolumn{6}{|c|}{ Between area variance covariance matrix } \\
\hline Prosocial & $0.022(0.011)$ & & & & \\
\hline Emotional & $-0.013(0.007)$ & $0.032(0.008)$ & & & \\
\hline Conduct & $-0.023(0.009)$ & $0.031(0.008)$ & $0.054(0.015)$ & & \\
\hline Hyperactivity & $-0.023(0.011)$ & $0.033(0.009)$ & $0.053(0.014)$ & $0.068(0.002)$ & \\
\hline Peer & $-0.011(0.007)$ & $0.028(0.006)$ & $0.029(0.008)$ & $0.029(0.010)$ & $0.031(0.009)$ \\
\hline \multicolumn{6}{|c|}{ Between children variance covariance matrix } \\
\hline Prosocial & $3.299(0.043)$ & & & & \\
\hline Emotional & $-0.200(0.024)$ & $1.915(0.024)$ & & & \\
\hline Conduct & $-1.223(0.035)$ & $0.700(0.026)$ & $3.799(0.048)$ & & \\
\hline Hyperactivity & $-1.228(0.041)$ & $0.663(0.030)$ & $2.043(0.045)$ & $5.079(0.067)$ & \\
\hline Peer & $-0.750(0.027)$ & $0.587(0.020)$ & $0.665(0.028)$ & $0.697(0.033)$ & $2.081(0.028)$ \\
\hline \multicolumn{6}{|l|}{ Model 14} \\
\hline \multicolumn{6}{|c|}{ Between area variance covariance matrix } \\
\hline Prosocial & $0.005(0.017)$ & & & & \\
\hline Emotional & $-0.005(0.009)$ & $0.008(0.009)$ & & & \\
\hline Conduct & $-0.006(0.014)$ & $0.015(0.01)$ & $0.029(0.02)$ & & \\
\hline Hyperactivity & $-0.004(0.016)$ & $0.029(0.012)$ & $0.011(0.018)$ & $0.024(0.027)$ & \\
\hline Peer & $-0.011(0.011)$ & $0.007(0.008)$ & $0.003(0.011)$ & $-0.003(0.013)$ & $0.023(0.013)$ \\
\hline \multicolumn{6}{|c|}{ Between children variance covariance matrix } \\
\hline Prosocial & $2.848(0.056)$ & & & & \\
\hline Emotional & $-0.093(0.029)$ & $1.468(0.028)$ & & & \\
\hline Conduct & $-0.949(0.043)$ & $0.375(0.029)$ & $2.992(0.058)$ & & \\
\hline Hyperactivity & $-0.871(0.051)$ & $0.358(0.036)$ & $1.478(0.054)$ & $4.304(0.085)$ & \\
\hline Peer & $-0.500(0.033)$ & $0.384(0.023)$ & $0.361(0.033)$ & $0.387(0.04)$ & $1.668(0.034)$ \\
\hline
\end{tabular}

significantly associated with any externalizing psychopathology (although it was positively associated with emotional symptoms and peer problems and negatively associated with prosocial behavior) in the full model (Model 14), it buffered the effect of proximal adverse life events on conduct problems. Similarly, although non-verbal ability was not significantly associated with any internalizing psychopathology (although it was positively related to prosocial behavior and negatively related to both conduct problems and hyperactivity) in the full model (Model 14), it buffered the effect of proximal risk on conduct problems but also on emotional symptoms. Table 4 shows the model summary for specific psychopathology, and Table 5 shows the results of Model 17. 
Table 4 Model summary (specific psychopathology)

\begin{tabular}{|c|c|}
\hline Models & Specification \\
\hline Model 10 & Design variables \\
\hline Model 11 & Model $10+$ area random effect \\
\hline Model 12.1 & Model $11+$ proximal family risk \\
\hline Model 12.2 & Model $11+$ distal family risk \\
\hline Model 12.3 & Model $11+$ area risk \\
\hline Model 13 & Model $11+$ area risk + measures of family risk (proximal and distal family risk) \\
\hline Model 14 & $\begin{array}{l}\text { Model } 13+\text { family level fixed effects (family structure, mother's qualifications, } \\
\text { mother's social class, mother's psychological distress) + child level fixed effects } \\
\text { (age, gender) + moderator variables (mood, regularity, adaptability, non-verbal } \\
\text { ability, verbal ability, developmental delay, negligent parenting) }\end{array}$ \\
\hline Model 15.1 & Model $14+$ interaction between proximal family risk and distal family risk \\
\hline Model 15.2 & Model $14+$ interaction between proximal family risk and area risk \\
\hline Model 15.3 & Model $14+$ interaction between distal family risk and area risk \\
\hline Model 16.1 & Model $14+$ interaction between proximal family risk and mood \\
\hline Model 16.2 & Model $14+$ interaction between proximal family risk and regularity \\
\hline Model 16.3 & Model $14+$ interaction between proximal family risk and adaptability \\
\hline Model 16.4 & Model $14+$ interaction between proximal family risk and non-verbal ability \\
\hline Model 16.5 & Model $14+$ interaction between proximal family risk and verbal ability \\
\hline Model 16.6 & Model $14+$ interaction between proximal family risk and developmental delay \\
\hline Model 16.7 & Model $14+$ interaction between proximal family risk and parenting \\
\hline Model 17 & $\begin{array}{l}\text { Model } 14+\text { interaction between proximal family risk and developmental } \\
\text { delay }+ \text { interaction between proximal family risk and non-verbal ability }\end{array}$ \\
\hline
\end{tabular}

\section{Discussion}

This study was carried out to test the hypothesis that for preschool children's behavioral and emotional adjustment in the face of contextual risk, factors promoting resilience should be located in the cognitive domain. Indeed, this study showed that neither parenting nor temperament buffered the effect of contextual risk on young children's emotional and behavioral adjustment. However, non-verbal ability moderated the effect of cumulative proximal risk on both emotional symptoms and conduct problems.

In testing this hypothesis, we measured and modeled contextual risk appropriately and we, therefore, also extended in several ways prior work on contextual risk effects on children's behavioral and emotional adjustment. First, assessing with well-validated measures family contextual risk, we showed that, although the number of family adversities experienced in the child's first year (distal family risk) did predict broad and externalizing psychopathology, the number of proximal (i.e., in the second and third year) family adversities (proximal family risk) predicted both broad and externalizing but also internalizing psychopathology. Second, we showed that the most parsimonious model was the model that included cumulative rather than specific family risk. Taken together, these findings highlight the importance of proximal family risk in predicting both broad and specific psychopathology, and the importance of considering family risk accumulation rather than specificity in predicting psychopathology in young children. Third, we showed that although the effect of area and the effect of area level contextual risk were significant on broad psychopathology even after distal and proximal family risk were controlled for, they became nonsignificant after adjusting for maternal socioeconomic status and mental health. This finding suggests that the effect of area and the effect of area risk on child psychopathology operate via family characteristics. The effect of distal and proximal family risk, on the other hand, remained significant after adjusting for these maternal characteristics suggesting that family contextual risk predicts child psychopathology directly and independently, and not because it is associated with parental psychopathology or social class. In other words, the effect of family contextual risk on child psychopathology transcends social origins and genetic predispositions. Fourth and finally, by testing for the functional form of the effect of both area and family risk and for the interaction between the various types and levels of risk, we joined the few other researchers [4, 11-14] that have tested for 'threshold' models of multiple risk. Ours, however, was the first study to test such effects on young children's psychopathology.

Of course, the study's limitations should also be acknowledged. For example, the children of the final study sample were clearly more privileged than those not included in the final study sample. However, in our modelling framework data were treated under the MAR assumption. 
Table 5 Specific psychopathology (Model 17)

\begin{tabular}{|c|c|c|c|c|c|}
\hline Predictors & $\begin{array}{l}\text { Prosocial } \\
\text { behavior } \\
\text { Coeff. (SE) }\end{array}$ & $\begin{array}{l}\text { Emotional } \\
\text { symptoms } \\
\text { Coeff. (SE) }\end{array}$ & $\begin{array}{l}\text { Conduct } \\
\text { problems } \\
\text { Coeff. (SE) }\end{array}$ & $\begin{array}{l}\text { Hyperactivity } \\
\text { Coeff. (SE) }\end{array}$ & $\begin{array}{l}\text { Peer problems } \\
\text { Coeff. (SE) }\end{array}$ \\
\hline Constant & $6.633(0.558)$ & $2.306(0.401)$ & $3.789(0.573)$ & $3.989(0.698)$ & $2.870(0.445)$ \\
\hline \multicolumn{6}{|l|}{ Stratum (Ref: England-advantaged) } \\
\hline England-disadvantaged & $-0.050(0.076)$ & $-0.032(0.054)$ & $0.017(0.078)$ & $0.078(0.094)$ & $0.181(0.063)$ \\
\hline England-ethnic & $0.375(0.139)$ & $0.128(0.099)$ & $0.038(0.146)$ & $0.076(0.176)$ & $0.259(0.117)$ \\
\hline Wales-advantaged & $0.033(0.118)$ & $-0.046(0.084)$ & $-0.213(0.122)$ & $0.118(0.146)$ & $-0.134(0.097)$ \\
\hline Wales-disadvantaged & $0.177(0.109)$ & $-0.044(0.079)$ & $0.154(0.114)$ & $0.237(0.136)$ & $-0.082(0.091)$ \\
\hline Scotland-advantaged & $-0.037(0.105)$ & $-0.058(0.074)$ & $-0.042(0.108)$ & $-0.042(0.129)$ & $-0.067(0.086)$ \\
\hline Scotland-disadvantaged & $-0.194(0.123)$ & $-0.079(0.088)$ & $0.141(0.128)$ & $0.139(0.153)$ & $0.062(0.101)$ \\
\hline Northern Ireland-advantaged & $0.024(0.134)$ & $-0.036(0.094)$ & $-0.232(0.136)$ & $-0.197(0.164)$ & $-0.187(0.108)$ \\
\hline Northern Ireland-disadvantaged & $0.154(0.133)$ & $-0.085(0.094)$ & $-0.134(0.137)$ & $-0.408(0.164)$ & $-0.082(0.108)$ \\
\hline Standardized IMD rank & $-0.037(0.035)$ & $-0.012(0.025)$ & $-0.028(0.036)$ & $0.077(0.043)$ & $-0.033(0.029)$ \\
\hline Proximal adverse life events & $-0.071(0.147)$ & $0.171(0.105)$ & $0.511(0.149)$ & $0.134(0.181)$ & $0.073(0.118)$ \\
\hline Distal adverse life events & $0.025(0.030)$ & $0.031(0.021)$ & $0.096(0.030)$ & $0.132(0.037)$ & $0.035(0.024)$ \\
\hline \multicolumn{6}{|l|}{ Family structure (Ref: two natural parents) } \\
\hline Natural mother only & $0.079(0.077)$ & $0.079(0.054)$ & $0.170(0.078)$ & $0.110(0.095)$ & $0.082(0.061)$ \\
\hline Other & $-0.092(0.060)$ & $-0.131(0.459)$ & $-0.745(0.617)$ & $-1.340(0.833)$ & $0.425(0.525)$ \\
\hline \multicolumn{6}{|l|}{ Mother's NS-SEC (Ref: high managerial/professional) } \\
\hline Low managerial/professional & $0.002(0.088)$ & $-0.065(0.062)$ & $-0.064(0.089)$ & $0.120(0.108)$ & $0.039(0.070)$ \\
\hline Intermediate & $0.015(0.099)$ & $-0.136(0.069)$ & $0.069(0.099)$ & $0.280(0.121)$ & $-0.082(0.078)$ \\
\hline Small emp and self-employed & $0.109(0.138)$ & $-0.159(0.097)$ & $-0.243(0.138)$ & $-0.173(0.168)$ & $-0.035(0.109)$ \\
\hline Low supervisory and technical & $0.123(0.126)$ & $-0.123(0.089)$ & $0.056(0.127)$ & $0.445(0.156)$ & $0.174(0.100)$ \\
\hline Semi routine & $-0.107(0.104)$ & $-0.037(0.073)$ & $0.347(0.104)$ & $0.332(0.128)$ & $0.082(0.083)$ \\
\hline Routine & $0.017(0.116)$ & $0.001(0.082)$ & $0.173(0.117)$ & $0.276(0.142)$ & $0.173(0.092)$ \\
\hline \multicolumn{6}{|l|}{ Mother's highest qualifications (Ref: level 5) } \\
\hline Level 4 & $-0.100(0.115)$ & $-0.050(0.081)$ & $-0.096(0.117)$ & $-0.035(0.140)$ & $-0.120(0.094)$ \\
\hline Level 3 & $-0.048(0.124)$ & $-0.004(0.088)$ & $0.010(0.126)$ & $0.345(0.152)$ & $0.067(0.101)$ \\
\hline A/AS Level & $-0.108(0.125)$ & $0.022(0.088)$ & $-0.058(0.127)$ & $0.272(0.153)$ & $0.056(0.102)$ \\
\hline Level 2 & $-0.111(0.117)$ & $0.096(0.083)$ & $0.170(0.119)$ & $0.495(0.143)$ & $0.141(0.096)$ \\
\hline Level 1 & $-0.092(0.134)$ & $0.311(0.095)$ & $0.400(0.136)$ & $0.722(0.165)$ & $0.229(0.110)$ \\
\hline Mother's psychological distress & $-0.032(0.014)$ & $0.085(0.010)$ & $0.158(0.015)$ & $0.159(0.018)$ & $0.064(0.012)$ \\
\hline Girl & $0.306(0.045)$ & $-0.009(0.032)$ & $-0.143(0.046)$ & $-0.505(0.056)$ & $-0.152(0.036)$ \\
\hline Age & $0.051(0.121)$ & $0.109(0.088)$ & $0.013(0.124)$ & $0.580(0.152)$ & $-0.066(0.097)$ \\
\hline Mood & $0.064(0.007)$ & $-0.025(0.005)$ & $-0.053(0.007)$ & $-0.051(0.009)$ & $-0.017(0.006)$ \\
\hline Regularity & $0.003(0.008)$ & $-0.019(0.006)$ & $-0.031(0.009)$ & $-0.043(0.010)$ & $-0.035(0.007)$ \\
\hline Adaptability & $0.017(0.007)$ & $-0.050(0.005)$ & $-0.020(0.007)$ & $-0.015(0.008)$ & $-0.039(0.005)$ \\
\hline Non-verbal ability & $0.009(0.003)$ & $-0.000(0.002)$ & $-0.011(0.003)$ & $-0.016(0.004)$ & $-0.002(0.003)$ \\
\hline Verbal ability & $0.021(0.007)$ & $-0.016(0.005)$ & $-0.014(0.007)$ & $-0.033(0.008)$ & $-0.020(0.005)$ \\
\hline Developmental delay & $-0.076(0.015)$ & $0.023(0.011)$ & $0.029(0.015)$ & $0.009(0.019)$ & $0.026(0.012)$ \\
\hline Negligent parenting & $-0.095(0.015)$ & $0.005(0.011)$ & $0.015(0.015)$ & $0.035(0.019)$ & $0.028(0.012)$ \\
\hline Proximal adverse life events by developmental delay & $0.004(0.008)$ & $-0.004(0.005)$ & $-0.017(0.008)$ & $0.004(0.010)$ & $-0.002(0.006)$ \\
\hline Proximal adverse life events by non-verbal ability & $-0.001(0.001)$ & $-0.002(0.001)$ & $-0.002(0.001)$ & $-0.002(0.002)$ & $0.001(0.001)$ \\
\hline
\end{tabular}

Our study findings have important implications for the study of both contextual risk and resilience as they suggest that, even among young children, contextual factors that impede adjustment are proximal rather than distal, and should be modeled cumulatively and located in the family rather than the area, and, importantly for the study of resilience, that factors that promote adjustment in the face of such contextual risk should be located among individual 
attributes pertaining specifically to cognitive strengths. Future studies should test this as well as the generaliseability of these findings in older child samples as the adverse characteristics of a neighborhood might impact differently upon the psychopathology of older children.

Acknowledgments This program of research was supported by a grant from the British Academy to the first two authors. The authors are grateful to Jon Johnson, Rachel Rosenberg, and Tina Roberts for their help with the construction of the dataset.

\section{References}

1. Rutter M (1979) Protective factors in children's responses to stress and disadvantage. In: Kent MW, Rolf JE (eds) Primary prevention of psychopathology: III. Promoting social competence and coping in children. University Press of New England, Hanover, pp 49-74

2. Evans GW (2003) A multimethodological analysis of cumulative risk and allostatic load among rural children. Dev Psychol 39:924-933

3. Burchinal M, Roberts J, Hooper S, Zeisel S (2000) Cumulative risk and early cognitive development: a comparison of statistical risk models. Dev Psychol 36:793-807

4. Flouri E, Kallis C (2007) Adverse life events and psychopathology and prosocial behavior in late adolescence: testing the timing, specificity, accumulation, gradient, and moderation of contextual risk. J Am Acad Child Adolesc Psychiatry 46:16511659

5. Deater-Deckard K, Dodge KA, Bates JE, Pettit GS (1998) Multiple risk factors in the development of externalizing behavior problems: group and individual differences. Dev Psychopathol 10:469-493

6. Atzaba-Poria N, Pike A, Deater-Deckard K (2004) Do risk factors for problem behaviour act in a cumulative manner? An examination of ethnic minority and majority children through an ecological perspective. J Child Psychol Psychiatry 45:707-718

7. Sameroff AJ, Seifer R, Baldwin A, Baldwin C (1993) Stability of intelligence from preschool to adolescence: the influence of social and family risk factors. Child Dev 64:80-97

8. Sheinkopf SJ, Lagasse LL, Lester BM et al (2007) Vagal tone as a resilience factor in children with prenatal cocaine exposure. Dev Psychopathol 19:649-673

9. Burchinal MR, Roberts JE, Zeisel SA, Rowley SJ (2008) Social risk and protective factors for African American children's academic achievement and adjustment during the transition to middle school. Dev Psychol 44:286-292

10. Ackerman BP, Brown ED, Izard CE (2004) The relations between persistent poverty and contextual risk and children's behavior in elementary school. Dev Psychol 40:367-377

11. Flaherty EG, Thompson R, Litrownik AJ et al (2006) Effect of early childhood adversity on child health. Arch Pediatr Adolesc Med 160:1232-1238

12. Gerard JM, Buehler C (2004) Cumulative environmental risk and youth problem behavior. J Marriage Fam 66:702-720

13. Morales JR, Guerra NG (2006) Effects of multiple context and cumulative stress on urban children's adjustment in elementary school. Child Dev 77:907-923

14. Simmons RG, Burgeson R, Carlton-Ford S, Blyth DA (1987) The impact of cumulative change in early adolescence. Child Dev 58:1220-1234
15. Leventhal T, Brooks-Gunn J (2000) The neighborhoods they live in: the effects of neighborhood residence on children and adolescent outcomes. Psychol Bull 126:309-337

16. McCulloch A, Joshi HE (2001) Neighborhood and family influences on the cognitive ability of children in the British National Child Development Study. Soc Sci Med 53:59-591

17. Ackerman BP, Izard CE, Schoff K, Youngstrom EA, Kogos J (1999) Contextual risk, caregiver emotionality, and the problem behaviors of six- and seven-year-old children from economically disadvantaged families. Child Dev 70:1415-1427

18. Tiet QQ, Bird HR, Davies M et al (1998) Adverse life events and resilience. J Am Acad Child Adolesc Psychiatry 37:1191-1200

19. Masten AS (2001) Ordinary magic: resilience processes in development. Am Psychol 56:227-238

20. Masten AS, Burt KB, Roisman GI, Obradovic J, Long JD, Tellegen A (2004) Resources and resilience in the transition to adulthood: continuity and change. Dev Psychopathol 16:1071-1094

21. Noble M, Wright G, Smith G, Dibben C (2006) Measuring multiple deprivation at the small area level. Environ Plann A 38:169-185

22. Brand AH, Johnson JH (1982) Note on reliability of the Life Events Checklist. Psychol Rep 50:1274

23. Coddington RD (1972) The significance of life events as etiologic factors in the diseases of children: I. A survey of professional workers. J Psychosom Res 16:7-18

24. Coddington RD (1972) The significance of life events as etiologic factors in the diseases of children: II. A study of a normal population. J Psychosom Res 16:205-213

25. Tiet QQ, Bird HR, Hoven CW et al (2001) Relationship between specific adverse life events and psychiatric disorders. J Abnorm Child Psychol 29:153-164

26. Goodman R (1994) A modified version of the Rutter Parent Questionnaire including extra items on children's strengths: a research note. J Child Psychol Psychiatry 35:1483-1494

27. Goodman R (1997) The Strengths and Difficulties Questionnaire: a research note. J Child Psychol Psychiatry 38:581-586

28. Goodman R (2001) Psychometric properties of the Strengths and Difficulties Questionnaire. J Am Acad Child Adolesc Psychiatry 40:1337-1345

29. Goodman R, Scott S (1999) Comparing the Strengths and Difficulties Questionnaire and the Child Behavior Checklist: is small beautiful? J Abnorm Child Psychol 27:17-24

30. Goodman R, Meltzer H, Bailey V (1998) The Strengths and Difficulties Questionnaire: a pilot study on the validity of the selfreport version. Eur Child Adolesc Psychiatry 7:125-130

31. Rutter MJ, Tizard J, Whitmore K (1970) Education, health and behaviour. Longman, London

32. Rodgers B, Pickles A, Power C, Collishaw S, Maughan B (1999) Validity of the Malaise Inventory in general population samples. Soc Psychiatry Psychiatr Epidemiol 34:333-341

33. Frankenburg WK, Dodds JB (1967) Denver Developmental Screening Test. J Paediatr 71:181-191

34. Carey W, McDevitt S (1978) Revision of the Infant Temperament Questionnaire. Pediatrics 61:735-739

35. Carey W, McDevitt S (1995) Coping with children's temperament: a guide for professionals. Basic Books, New York

36. Elliot CD (1983) British Ability Scales. NFER-Nelson, Windsor

37. Bracken BA (1998) Bracken Basic Concept Scale-revised. The Psychological Corporation, Harcourt Brace and Company, San Antonio

38. Goldstein H (2003) Multilevel statistical models, 3rd edn. Arnold, London

39. Schwarz G (1978) Estimating the dimension of a model. Ann Stat $6: 461-464$ 